\title{
Preface to WISM 2011
}

The international workshop on Web Information Systems Modeling (WISM) aims to uncover the most recent developments in the field of model-driven design of Web Information Systems (WIS). This is the eighth edition of the workshop, after seven successful editions organized in Vancouver (2010), Amsterdam (2009), Barcelona (2008), Trondheim (2007), Luxembourg (2006), Sydney (2005), and Riga (2004).

The extended abstract of the invited talk by de Oliveira et al. proposes concepts for Social Network Analysis to be applied for online Academic Social Networks. More specifically, it investigates the network analysis, work dissemination, and partner recommendation in an academic context. The social network based on co-author relationships among researchers is used as an example.

The first paper by McGinnes proposes research directions for handling data exchange between heterogeneous WIS. These research directions are developed around the idea of building a basic ontology containing a simple set of shared concepts. The author argues that knowing the types of the shared concepts is not enough to ensure WIS interoperability, as WIS need be able to programmatically handle these types.

The second paper by Aguilar et al. suggests a solution for selecting the appropriate WIS requirements in order to maximize the user satisfaction with respect to a prioritized list of non-functional requirements. The approach is based on detecting the Pareto front of candidate solutions and selecting the ones that ensure the fulfillment of the highly ranked non-functional requirements. For demonstration purposes the authors use the requirements of an online Conference Management System represented using the $i^{*}$ framework.

The third paper by Boukhebouze et al. proposes UI-BPEL, an extension of BPEL that allows the modeling of user interaction (providing, selecting, and getting data by the user) in Web service composition. The main of goal of UI-BPEL is to allow the generation of user interfaces based on the described user interactions. This process is composed of two steps: the generation of an abstract user interface independent of any interaction modality and computing platform, and the generation of a concrete user interface based on the abstract user interface that is adapted to the user specific context.

The fourth paper by Bianchini et al. describes an approach for rapid WIS development by composing existing Web APIs. The composition is based on selection patterns that exploit functional similarities and coupling criteria. For this purpose Web APIs are annotated using existing lightweight semantic models extended with semantically annotated events.

We do hope that the previous synopsis has triggered the reader's interest to have a closer look at the workshop proceedings. Last, we would also like to thank all the authors, reviewers, and participants for their input and support for the workshop. 